\title{
Structural challenges to implementation of early intervention of the bio-psychosocial model
}

Nikki Brouwers

The Interact Group, North Sydney NSW 2060, Australia. nikki@interactgroup.com.au

Early intervention of the biopsychosocial model as a framework to achieve sustainable employment outcomes following injury, illness or disease is well documented in the International research. However in some areas of disability management the term early intervention is consistently applied exclusively within the medical model with early access to treatment and diagnostic tools.

However in Australia, whilst the knowledge of the Health benefits of work consensus statement is gaining traction, we remain lagging in effective implementation of early intervention models of the biopsychosocial model to return to work and return to life. This paper explores the structural challenges associated with successful implementation of the model and provides International learnings and applications for consideration and adaption to the Australian context. Further this paper will provide learnings from a pilot program being run in NSW that has been successful in the early triage and referral into a structured Return to Work program that is achieving strong results. 